\title{
Wearable Antenna for Power Efficient On-Body and Off-Body Communications
}

\author{
Mohammad Monirujjaman Khan1,2, Md. Azizur Rahman33, Md. Abu Talha1, \\ Tarannum Mithila1 \\ ${ }^{1}$ Electronics and Telecommunication Engineering, University of Liberal Arts Bangladesh, Dhaka, Bangladesh \\ ${ }^{2}$ School of Electronic Engineering and Computer Science, Queen Mary University of London, London, UK \\ ${ }^{3}$ Department of Computer \& Information Technology, London School of Technology, London, UK \\ Email: monirkhan.qmul@gmail.com
}

Received 1 July 2014; revised 28 July 2014; accepted 13 August 2014

Copyright (C) 2014 by authors and Scientific Research Publishing Inc.

This work is licensed under the Creative Commons Attribution International License (CC BY).

http://creativecommons.org/licenses/by/4.0/

(C) (i) Open Access

\section{Abstract}

A novel dual-band and diverse radiation pattern antenna is proposed for power efficient on-body and off-body communications intended for various applications in healthcare and sport monitoring. The antenna is dual band at $2.45 \mathrm{GHz}$ (ISM band) with omnidirectional radiation pattern over the body surface to communicate power efficiently with other co-located body worn devices and at 1.9 GHz (PCS band), it has directive radiation pattern towards off the body to communicate from on-body device to off-body devices. The free space and on-body performances of the antenna are investigated by both simulation and experiment. The antenna shows very good on-body radiation efficiency of $58 \%$ at $2.45 \mathrm{GHz}$ and $61 \%$ at $1.9 \mathrm{GHz}$. Good on-body gain is noticed at both frequency bands. Results show that the gain of the proposed antenna increases by $4.7 \%$ at $2.45 \mathrm{GHz}$ and $3.2 \%$ at $1.9 \mathrm{GHz}$ when placed on the body.

\section{Keywords}

Dual Band, Diverse Radiation Pattern, Power Efficient On-Body Communications, Power Efficient Off-Body Communications, Body-Centric Wireless Communications

\section{Introduction}

Body-centric wireless communications (BCWCs) are a central point in the development of fourth generation mobile communications [1]-[3]. In common healthcare monitoring scenarios, it is very important for the antenna to radiate over the body surface omni-directionally and also directive towards off the body units in order to get the best on-body and off-body radio channel performances i.e. minimising the link loss to ensure power effi- 
ciency. Body-centric wireless devices need to offer low power consumption in order to extend the battery life of the body worn devices and also need to provide power efficient and reliable on-body and off-body communications. Recently there have been growing interest and research development both in academia and industry in designing narrowband body worn antennas [4]-[10]. Hall et al. [4] [5] have undertaken extensive studies on narrowband antennas $(2.45 \mathrm{GHz})$ for on-body communication. In [6] a dual band button antenna for WLAN (2.45 $\mathrm{GHz}, 5.2 \mathrm{GHz}$ ) applications was presented by Batchelor. The antenna shows same radiation characteristics at both frequency bands. Alomainy et al. [7]-[9] presented various wearable antennas in the $2.4 \mathrm{GHz}$ (ISM band) for on-body communications. Furthermore, in [10], Scanlon et al. presented a set of higher mode microstrip patch antennas operating at $2.45 \mathrm{GHz}$ for over the body surface communications. Antenna radiation pattern influences the on/off-body radio channels performance. Antenna with omnidirectional radiation pattern over the body surface improves the path gain for the on-body links while antenna with directive off-body radiation pattern improves the path gain for off-body channels.

However, to the author knowledge nothing has been performed in designing of antenna for power efficient on-body and off-body communications with different radiation modes at different frequencies. In this paper, a dual band and diverse radiation pattern antenna is proposed for power efficient and reliable cooperative on-body and off-body communications. The free space and on-body performance parameters of the proposed dual band and diverse radiation pattern antenna are investigated by both simulation and experiment. The rest of the paper is organized as follows; Section 2 illustrates the antenna design, Section 3 presents result and analysis, and finally Section 4 draws the main conclusion.

\section{Antenna Design}

Figure 1 and Figure 2(b) show the schematic diagram and fabricated version of the proposed dual band and di verse radiation pattern antenna. The antenna is modelled on FR4 substrate with a thickness of $1.57 \mathrm{~mm}$ and a relative permittivity of 4.6. There is a full ground plane at the back of the substrate with the size of $60 \times 60 \mathrm{~mm}$. The antenna contains two radiating elements, a disk loaded Monopole and circular Patch. The diameter of the top disk and lower patch is 39 and $34 \mathrm{~mm}$ respectively. Table 1 shows the dimensions of the antenna components designed. The antenna is excited using microstrip line feed connected with the circular patch printed on the substrate. The upper and lower disks are connected by placing a small ring with a diameter of $5 \mathrm{~mm}$ and a height of $2.23 \mathrm{~mm}$ on the lower disk. The inner ring works on the same principle as a Monopole, and by placing the disk on the top the size has been reduced which otherwise would radiate at much higher frequency. The lower circular disk works on the same principle as a patch. For the dual band and diverse radiation pattern antenna, the upper resonance frequency depends on the size of the inner ring and also on the size of top disk but the lower frequency depends on the diameter of the lower Patch and the size of the top disk.

Both disks were aligned asymmetrically for optimum impedance matching and radiation performance. In this structure, the top loaded disk Monopole structure is to achieve the resonance at $2.45 \mathrm{GHz}$ with omnidirectional radiation pattern over the body surface to communicate with other body worn devices whereas the lower circular patch on the FR4 board is for $1.9 \mathrm{GHz}$ with off the body directive radiation mode to communicate power-effi-

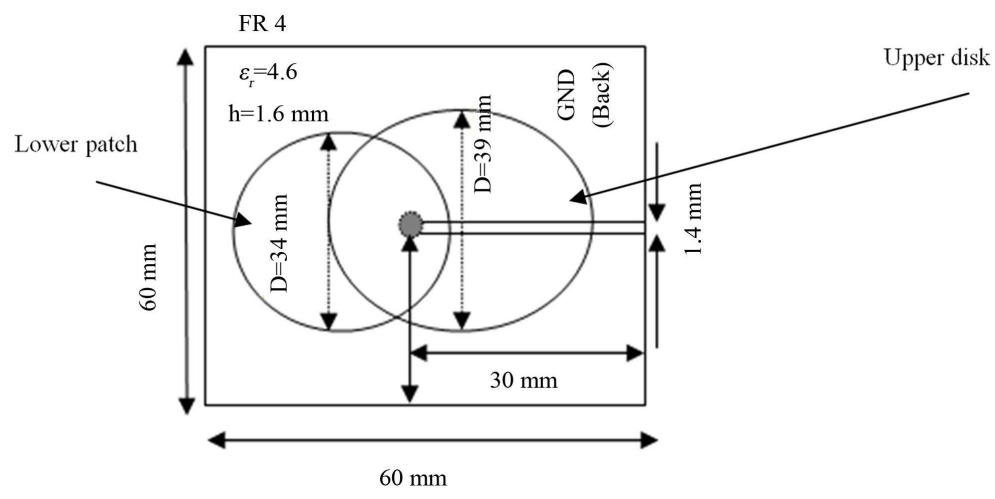

Figure 1. Schematic diagram of the dual band and diverse radiation pattern antenna. 


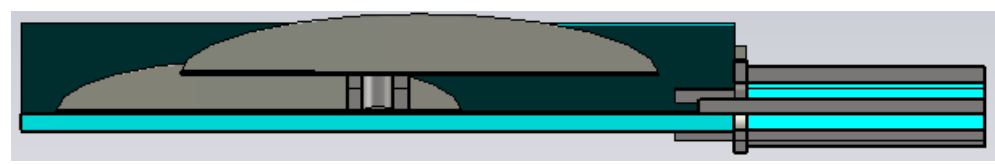

(a)

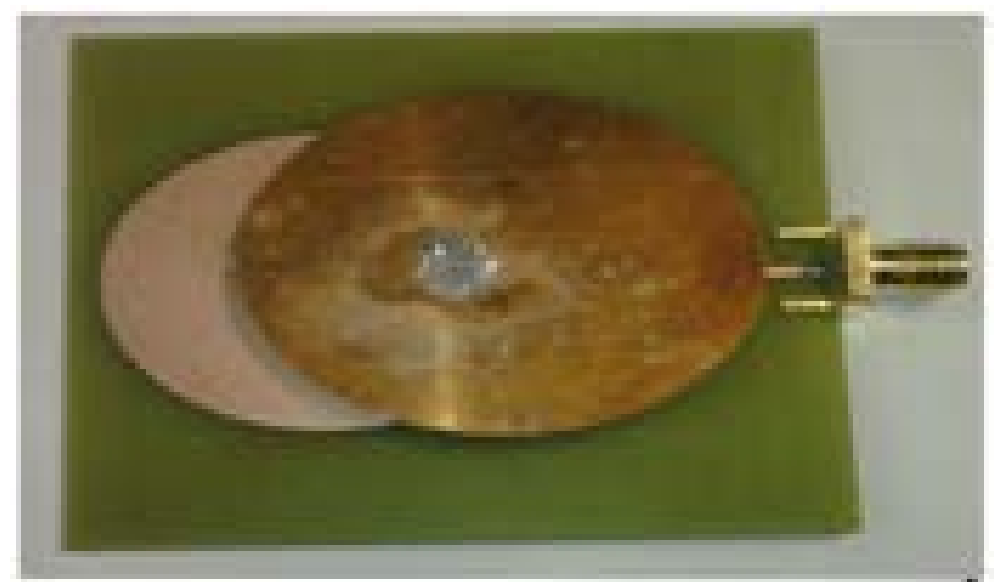

(a)

Figure 2. (a) Cross sectional view of the proposed dual band and diverse radio pattern antenna; (b) Fabricated antenna.

Table 1. Dimensions of the dual band and diverse radiation pattern antenna components.

\begin{tabular}{cc}
\hline Components & Unit (mm) \\
\hline Top disk diameter & 39 \\
Lower patch diameter & 34 \\
Height of inner ring & 2.23 \\
Ground plane size & $60 \times 60$ \\
\hline
\end{tabular}

ciently from on-body device to off-body units. Fundamental patch antennas have radiation characteristics suitable for the off-body channel, that is maximum gain normal to the patch surface [11] in the off-body direction thus improving path gain.

\section{Results and Analysis}

The antenna is simulated both in free space and on the human phantom (ground plane 4 mm away from right chest) using CST microwave studio ${ }^{\mathrm{TM}}$. The human body employed is the commonly available detailed multilayer model (Figure 3) namely the "visible male model” developed by the US Air Force [12]. The resolution of the model applied is $4 \mathrm{~mm}$ with the electrical properties of human tissues defined at $2.45 \mathrm{GHz}$ and $1.9 \mathrm{GHz}$, respectively, for all organs and tissues used including heart, lungs, muscle, fat, skin, etc. [13] [14]. The on-body performances of the antenna were measured on the real human body ( $4 \mathrm{~mm}$ away from right chest). Dual band characteristic is shown in Figure 4. Slight variation of the free space simulated and measured resonances are are noticed which can be due to fabrication perfectness. When the dual band and diverse radiation pattern antenna is measured on the body, a very slight frequency detuning from free space resonance $(0.30 \%)$ is observed for the upper frequency band whereas for the lower frequency band there is no frequency detuning. Simulation results show slight higher detuning of resonance frequency as compare to measured. The antenna shows good on-body bandwidth (at $-10 \mathrm{~dB} \mathrm{~S}_{11}$ ) of $2.40 \%$ for upper frequency band and $1.8 \%$ for the lower band.

The bandwidth of upper frequency band is close to the required bandwidth (3.2\%) of ISM band; however, the bandwidth of upper frequency can be increased by increasing the thickness of the inner ring. The simulated free space gain of the DBDM antenna is $4.0 \mathrm{dBi}$ for $2.45 \mathrm{GHz}$ and $4.3 \mathrm{dBi}$ for $1.9 \mathrm{GHz}$ which increases by $4.7 \%$ for 


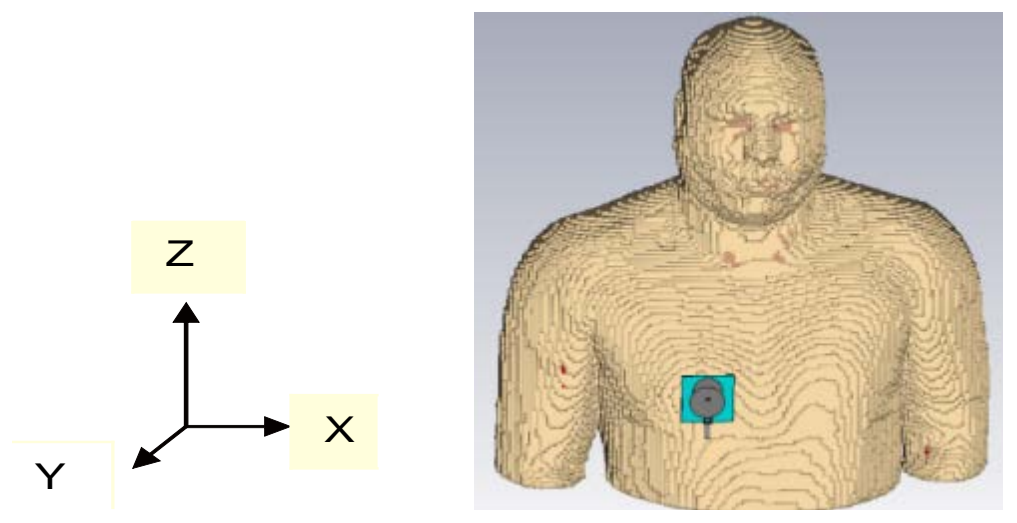

Figure 3. Human phantom used for simulation showing antenna location and orientation on the body.

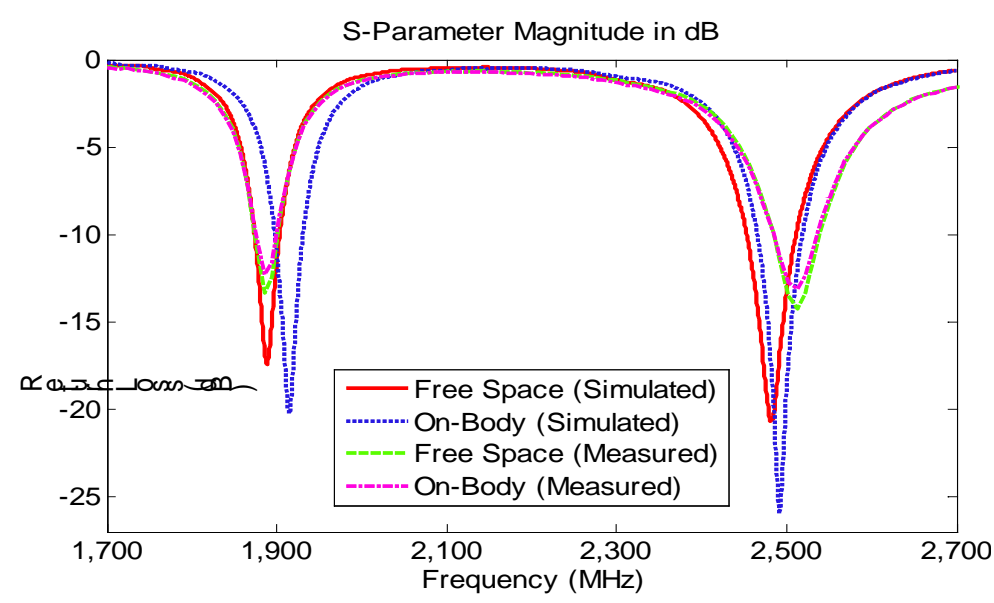

Figure 4. Simulated and measured free space and on-body return loss curves of the proposed dual-band and diverse radiation pattern antenna.

the upper band and 3.2\% for the lower band when placed on the body. The antenna shows very good on-body radiation efficiency of $58 \%$ for $2.45 \mathrm{GHz}$ and $61 \%$ for $1.9 \mathrm{GHz}$.

The antenna is proposed to be used in BCWCs where communication is necessary both to the devices onbody and to the external off-body network nodes. With the antenna mounted on the body, it is observed that at $1.9 \mathrm{GHz}$ the radiation is directive towards off the body (Figure 3); see Figure 5(a) and Figure 5(b). It has a very good coverage in the forward direction of the user body with the $Y$ direction exhibiting the maximum gain. This is ideal for power-efficient communication by improving path gain of the radio link from the body worn base station to the off-body devices. The lower printed patch element radiates like normal patch antenna with the maximum radiation to the $\mathrm{Y}$ direction (toward the off-body) which gives maximum gain in that direction. Due to the presence of human body, at lower frequency band, loss in back lobe of the radiation pattern is noticed both in measurement and simulation.

At $2.45 \mathrm{GHz}$, the antenna radiates omnidirectional to the user surface in the XZ plane (Figure 3) with maximum gain at this plane; see Figure 6(a). When the antenna is body worn, there is also omnidirectional radiation in the $\mathrm{XZ}$ plane with slight loss in the simulation results but in measurement, the radiation looks nearly as stable as free space. For a user that needs to wear various sensors integrated with antenna system on different places of the body in order to read the vital electrical signs of the human body, power efficient communication is important from each node as well as in the human worn base controller. For this scenario, this kind of radiation pattern which is omnidirectional over the body surface with maximum gain is very useful to establish power-efficient on-body communications by improving the path gain for on-body radio links. In the YZ plane, at $2.45 \mathrm{GHz}$, the DBDM shows directive radiation pattern with little null in the front beam as shown in Figure 6(b). 


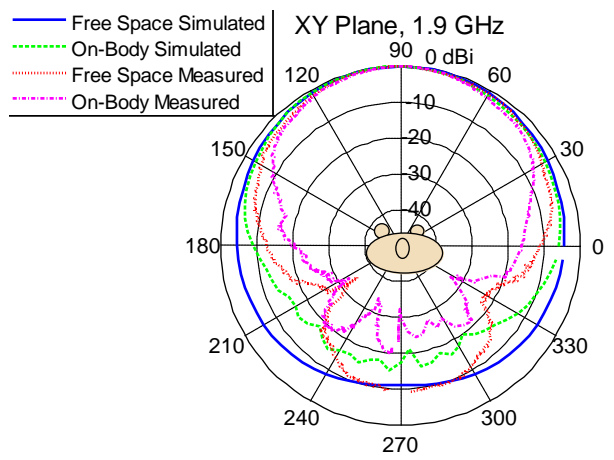

(a)

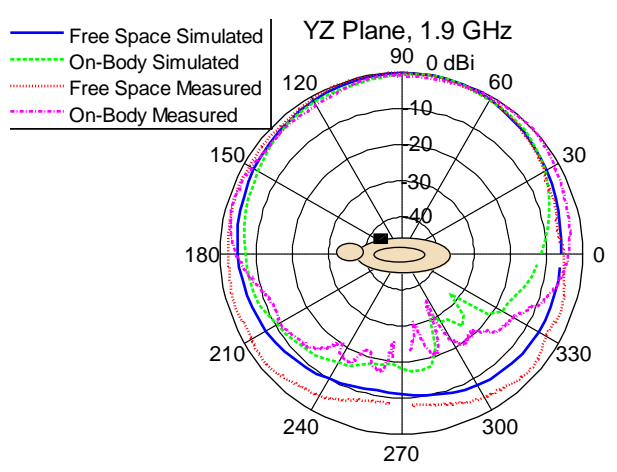

(b)

Figure 5. Simulated and measured free space and on-body radiation patterns of the proposed dual-band and diverse radiation pattern antenna (a) XY plane at $1.9 \mathrm{GHz}$ and (b) YZ plane at $1.9 \mathrm{GHz}$.

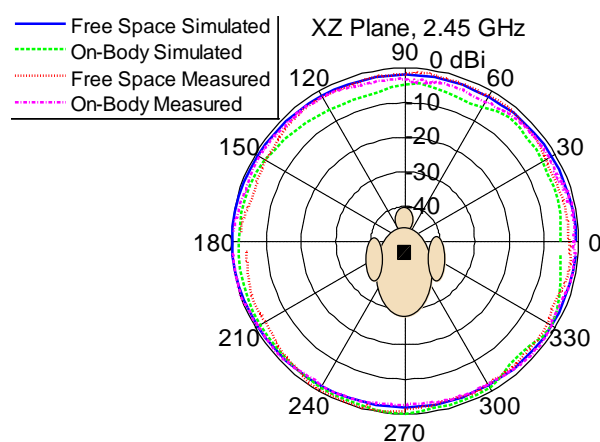

(a)

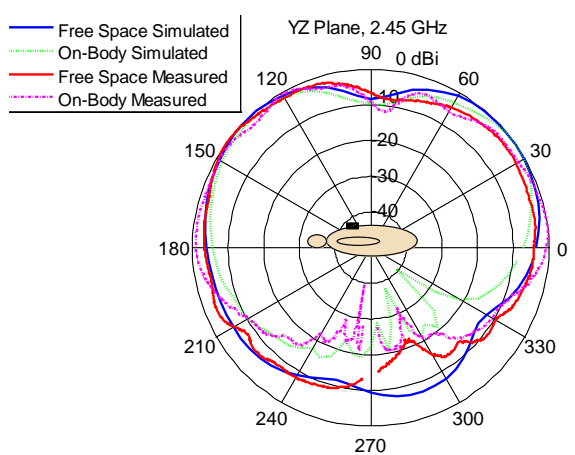

(b)

Figure 6. Simulated and measured free space and on-body radiation patterns of the proposed dual-band and diverse radiation pattern antenna (a) XZ plane at $2.45 \mathrm{GHz}$ and (b) YZ plane at $2.45 \mathrm{GHz}$.

\section{Conclusion}

A novel dual band and diverse radiation pattern antenna is proposed for power efficient on-body and off-body communications intended for applications in healthcare and sport monitoring. The human body effects on the performance of the antenna have been studied. The antenna shows omnidirectional radiation performances along the body surface at $2.45 \mathrm{GHz}$ for on-body communication and directive radiation pattern at $1.9 \mathrm{GHz}$ for communications with off-body devices. The antenna shows very good on-body performances in terms of radiation, gain, efficiency and frequency detuning. The antenna is conformal and contains only single microstrip feed. It will be a very good candidate for future BCWCs in order to establish power-efficient and reliable cooperative on-body and off-body communications.

\section{Acknowledgements}

The authors of this paper would like to thank John Dupuy for his help with the antenna fabrication. The authors also would like to thank Sanjoy Mazumdar for his help during the measurement.

\section{References}

[1] Hall, P.S. and Hao, Y. (2006) Antennas and Propagation for Body-Centric Communications. 1st Edition, Artech House, London and Boston. http://dx.doi.org/10.1049/ic:20070540

[2] Presser, M., Brown, T., Goulianos, A., Stavrou, S. and Tafazolli, R. (2007) Body-Centric Context Aware Application Scenarios. Proceedings of the IET Seminar on Antennas and Propagation for Body-Centric Wireless Communications, London, 24-24 April 2007, 19-23. 
[3] Khan, M.M., Abbasi, Q.H., Liaqat, S. and Alomainy, A. (2012) Comparison of Two Measurement Techniques for UWB Off-Body Radio Channel Characterisation. Progress in Electromagnetics Research M, 27, 179-189. http://dx.doi.org/10.2528/PIERM11102810

[4] Hall, P.S., Hao, Y., Nechayev, Y.I., Alomainy, A., Constantinou, C.C., Parini, C.G., Kamruddin, M.R., Salim, T.Z., Hee, D.T.M., Dubrovka, R., Wadally, A., Song, W., Serra, A., Nepa, P., Gallo, M. and Bozzetti, M. (2007) Antennas and Propagation for On-Body Communication Systems. IEEE Antennas and Propagation Magazine, 49, 41-58. http://dx.doi.org/10.1109/MAP.2007.4293935

[5] Nechayev, Y.I., Hall, P.S. and Hu, Z.H. (2010) Characterisation of Narrowband Communication Channels On the Human Body at 2.45 GHz. IET Microwaves Antennas and Propagation, 4, 722-732. http://dx.doi.org/10.1049/iet-map.2009.0094

[6] Sanz-Izquierdo, B., Huang, F. and Batchelor, J.C. (2006) Covert Dual-Band Wearable Button Antenna. Electronics Letters, 42, 3-4. http://dx.doi.org/10.1049/el:20060482

[7] Alomainy, A., Hao, Y. and Pasveer, F. (2007) Numerical and Experimental Evaluation of a Compact Sensor Antenna for HealthCare Devices. IEEE Transactions on Medical Circuits and Systems, 1, 242-249. http://dx.doi.org/10.1109/TBCAS.2007.913127

[8] Alomainy, A., Hao, Y., Owadally, A., Parini, C.G., Hall, P.S. and Constantinou, C.C. (2007) Statistical Analysis and Performance Evaluation for On-Body Radio Propagation with Microstrip Patch Antennas. IEEE Transactions on Antenna and Propagation, 55, 245-248. http://dx.doi.org/10.1109/TAP.2006.888462

[9] Alomainy, A., Hao, Y. and Davenport, D.M. (2007) Parametric Study of Wearable Antennas Varying Distances from the Body and Different On-Body Positions. Proceedings of the IET Seminar on Antennas and Propagation for BodyCentric Wireless Communications, London, 24-24 April 2007, 84-89.

[10] Conway, G.A. and Scanlon, W.G. (2009) Antennas for over Body-Surface Communication at 2.45 GHz. IEEE Transactions on Antennas and Propagation, 57, 844-855. http://dx.doi.org/10.1109/TAP.2009.2014525

[11] Balanis, C.A. (2005) Antenna Theory Analysis and Design. 3rd Edition, John Wiley and Sons, Inc., Hoboken.

[12] Electronic Imaging: Board of Regents (1997) National Institute of Health National Library of Medicine USA. Bard of regents, Bethesda, Tech, Rep. NH 90-2197. http://www.brooks.af.mil/AFRL/HED/hedr

[13] Gabriel, C. and Gabriel, S. (1999) Compilation of the Dielectric Properties of Body Tissues at RF and Microwave Frequencies. http://www.brooks.af.mil/AFRL/HED/hedr/reports/dielectric/Title/Title.html

[14] Institute for Applied Physics (2014) Italian National Research Council, Calculation of the Dielectric Properties of Body Tissues. http://niremf.ifac.cnr.it/tissprop/ 